\title{
A simple scoring system to predict ambulation prognosis after hip fracture in the elderly
}

\author{
Tetsuo Hagino $\cdot$ Satoshi Ochiai $\cdot$ Masanori Wako \\ Eiichi Sato $\cdot$ Shingo Maekawa $\cdot$ Shinya Senga $\cdot$ \\ Hajime Sugiyama $\cdot$ Yoshiki Hamada
}

Published online: 20 June 2007

(C) Springer-Verlag 2007

\section{Erratum to: Arch Orthop Trauma Surg DOI 10.1007/s00402-007-0330-y}

The conclusion section of the abstract was rendered incorrectly.

The correct version is given here.

\section{Abstract}

Introduction We conducted a study on elderly patients with hip fracture to examine whether it is possible to predict the ambulation status of these patients upon hospital discharge.

Material and methods One hundred and eighty-six patients with femoral neck or trochanteric fracture, who were ambulant prior to fracture, were studied. Thirteen factors that may affect walking ability were selected and subjected to multivariate analysis.

Results Of 186 patients, 145 regained walking ability at discharge. Factors significantly affecting walking ability at discharge were (1) anemia, (2) dementia and (3) abnormal chest X-ray. Each patient was scored on the basis of the above factors $(1=$ yes, $0=$ no), and the total was used as the predictive score.
Conclusion A simple scoring system composed of these three factors was useful for the prediction of the ambulation status upon hospital discharge.

The online version of the original article can be found under doi:10.1007/s00402-007-0330-y.

T. Hagino $(\bowtie) \cdot$ S. Ochiai $\cdot$ M. Wako

Department of Orthopaedic Surgery, Kofu National Hospital,

11-35 Tenjin-cho, Kofu, Yamanashi 400-8533, Japan

e-mail: tmhagino@amber.plala.or.jp

E. Sato $\cdot$ S. Maekawa $\cdot$ S. Senga $\cdot$ H. Sugiyama $\cdot$ Y. Hamada

Department of Orthopaedic Surgery, Faculty of Medicine,

University of Yamanashi, Yamanashi, Japan 